Bundesgesundheitsbl -

Gesundheitsforsch - Gesundheitsschutz

2000 43:198-209 @ Springer-Verlag 2000

Leitthema: Gesundheitsbezogene Lebensqualität

U. Ravens-Sieberer

Universität Hamburg

\title{
Verfahren zur Erfassung der gesundheitsbezogenen Lebensqualität bei Kindern und Jugendlichen
}

\section{Ein Überblick}

\section{Zusammenfassung}

Während die gesundheitsbezogene Lebensqualität Erwachsener zunehmend erforscht ist, wird die Lebensqualität gesunder und chronisch kranker Kinder und Jugendlicher erst jüngst thematisiert. In der vorliegenden Arbeit werden in der Literatur publizierte Arbeiten zum Thema gesundheitsbezogene Lebensqualität von Kindern ausgewertet hinsichtlich Schwerpunkten und Methoden der Forschung. Der Überblick zeigt, dass empirische Arbeiten bisher rar sind, die vorhandenen Messinstrumente zur Lebensqualität von Kindern werden beschrieben. Verstärkte Forschungsaktivitäten sind notwendig, um die Erfassung der Lebensqualität von Kindern in der Prävention, den Gesundheitswissenschaften, in Therapieevaluation und Rehabilitation einsetzen zu können.

\section{Schlüsselwörter}

Kinder - Gesundheitsbezogene Lebensqualität · Erfassungsinstrumente ährend die Lebensqualitätsforschung als Ausdruck der Neuorientierung in den Gesundheitswissenschaften einen beispiellosen Boom erlebt, blieb die Auseinandersetzung mit dem Konstrukt Lebensqualität lange Zeit weitestgehend auf erwachsene Patienten beschränkt $[1,2]$. So nahmen zwar in den letzten zehn Jahren theoretische und empirische Artikel zum Thema Lebensqualität in der Medizin zu, diese bezogen sich allerdings vorwiegend auf die Entwicklung von Instrumentarien und deren Einsatz in Studien bei Erwachsenen und nicht bei Kindern [3].

Der Begriff der ,,gesundheitsbezogenen Lebensqualität" beschreibt in der psychologischen und medizinischen Terminologie ein Konstrukt, das wie auch z.B. Angst oder Intelligenz, nicht direkt beobachtet werden kann, sondern $\mathrm{zu}$ erschließen ist über konstituierende Komponenten. Eine operationale Definition der gesundheitsbezogenen Lebensqualität besagt, dass gesundheitsbezogene Lebensqualität ein multidimensionales Konstrukt ist, das körperliche, emotionale, mentale, soziale und verhaltensbezogene Komponenten des Wohlbefindes und der Funktionsfähigkeit aus Sicht der Patienten und/oder von Beobachtern beinhaltet $[4,5,6]$. Internationale Arbeiten zur Lebensqualität haben gezeigt, dass hinsichtlich dieser Komponenten weitgehende Übereinstimmung besteht, und dass sie möglicherweise kulturübergreifend als Universalien des
Erlebens und Verhaltens von Personen gelten können [7].

Innerhalb des medizinischen Versorgungssystems kann heute nicht nur die Lebensqualität von Erwachsenen, sondern auch von Kindern als wichtiges Zielkriterium bei der Evaluation von medizinischen Maßnahmen angesehen werden [8].

\section{"Die Erforschung der Lebens- qualität von Kindern ist auch deshalb von Bedeutung, da die Zahl der Kinder und Jugend- lichen mit chronischen Erkran- kungen zugenommen hat."}

Die Inanspruchnahme medizinischer Hilfen im Bereich der Prävention (z.B. Vorsorgeuntersuchungen), im Bereich der Therapie und im Bereich der Rehabilitation (z.B. nach Unfällen) wirkt sich nicht nur auf somatische, sondern auch auf emotionale und soziale Parameter aus, weswegen es nötig ist, die Verfassung von Kindern und Jugendlichen aus ihrer eigenen Sicht zu beleuchten. Die Erforschung der Lebensqualität von Kindern ist vor allem auch deshalb von Bedeutung, da die Zahl der Kinder und

U. Ravens-Sieberer

Abteilung für Medizinische Psychologie, Pav 69, Universität Hamburg, 20246 Hamburg 


\section{U. Ravens-Sieberer}

\section{Assessment of health related quality of life in children and adolescent}

\section{Summary}

While health-related quality of life of adults is increasingly researched, the quality of life of healthy and chronically ill children and adolescents has only recently been addressed. The present paper analyses published articles on children's health-related quality of life, identified from medical and psychological sources according to focus and methodology in research. The review shows that empirical work is short and describes available instruments for the assessment of quality of life in children. Increased research activities are necessary to include quality of life assessment of children in prevention, public health, treatment evaluation and rehabilitation.

\section{Key words}

Children · Health-related quality of life . Assessment instruments
Jugendlichen mit chronischen Erkrankungen (z.B. Asthma bronchiale) trotz eindrucksvoller Fortschritte in der Medizin zugenommen hat.

Obwohl medizinische Interventionen in vielen Fällen zu einer objektiven Verbesserung des Gesundheitszustandes der Kinder führen, konnte nachgewiesen werden, dass häufige Krankenhausaufenthalte, multiple Operationen und nicht zuletzt die unsichere Zukunft einen ungünstigen Einfluss auf die Entwicklung und den Krankheitsverarbeitungsprozess von Kindern haben können [9]. Die Frage, wie es den Kindern im Zusammenhang mit ihrem Gesundheitszustand und der Behandlung geht, ist für die Bewertung des Therapieerfolgs ebenso wichtig wie für die Frage, wie im individuellen Fall die Behandlung optimal gestaltet werden kann [10, 11].

\section{Stand der gesundheits- bezogenen Lebensqualitäts- forschung bei Kindern}

Seit der ersten Nennung des Begriffes Lebensqualität in der Medizin (1964) sind in der medizinischen Literatur bisher über 20 ooo Publikationen zu diesem Thema erschienen (Abb. 1), jedoch beziehen sich nur rund $13 \%$ dieser Arbeiten auf die Lebensqualität von Kindern. Datengrundlage für diesen Überblick der Forschungsarbeiten ist ein Literaturreview (Quality of life + Child) in der Datenbank MEDLINE von Januar 1998 bis Juni 1999 am Deutschen Instituts für Medizinische Dokumentation und Information (DIMDI) in Köln.
In der neueren Forschungslandschaft finden in der Pädiatrie, neben bisher vorherrschenden theoretisch-konzeptionellen Arbeiten, empirische Studien zunehmend mehr Eingang, wobei die Erhebungen an chronisch kranken Kindern am häufigsten vertreten sind [12]. Betrachtet man die untersuchten Krankheitsbilder in den Veröffentlichungen über Lebensqualität und Kinder näher, so lassen sich nur schwache Zusammenhänge zwischen Prävalenz und Untersuchungshäufigkeit von Krankheitsbildern feststellen. So sind z.B. Asthma und atopische Dermatitis (Neurodermitis) die häufigsten chronischen Krankheitsbilder im Kindesalter. Epidemiologische Untersuchungen in Deutschland geben eine Prävalenz von diagnostiziertem Asthma in Höhe von $4,5 \%$ und für asthmatypische Beschwerden bis zu $9 \%$ bei Kindern zwischen sechs und neun Jahren an [13]. Asthma bronchiale rangiert nur an dritter Stelle bezüglich der Anzahl publizierter Arbeiten zu Lebensqualität und Kinder (Abb. 2). Häufig untersucht sind - neben Krankheiten mit hoher Mortalitätsrate - Krankheiten, bei denen es zu krisenhaften Zuspitzungen z.T. lebensbedrohlichen Ausmaßes kommen kann, und Krankheiten, deren Behandlung mit einem hohen Aufwand an Kosten verbunden ist. Die Beschäftigung mit häufiger vorkommenden und weniger bedrohlichen Krankheiten oder Krankheiten, die nur zeitlich begrenzt sind, tritt dagegen in den Hintergrund. Befunde zur gesundheitsbezogenen Lebensqualität gesunder Kinder existieren kaum, und Ansätze diesbezüglicher Forschungen sind aus-

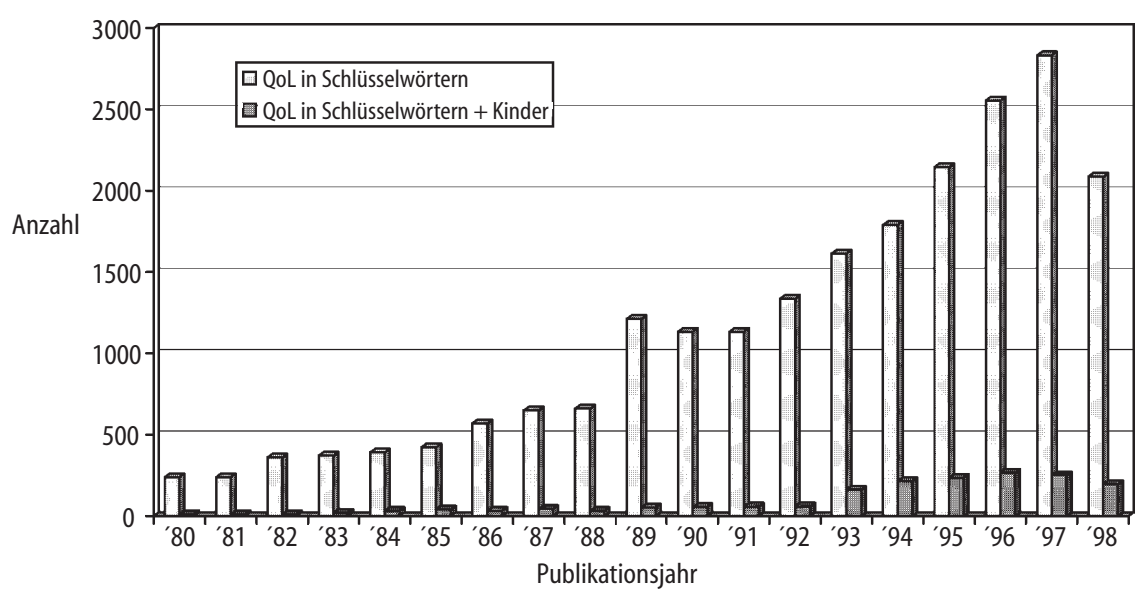

Abb. $1 \Delta$ Anzahl der Publikationen zum Thema Lebensqualität Erwachsener und Kinder (Medline 1980-1998) 


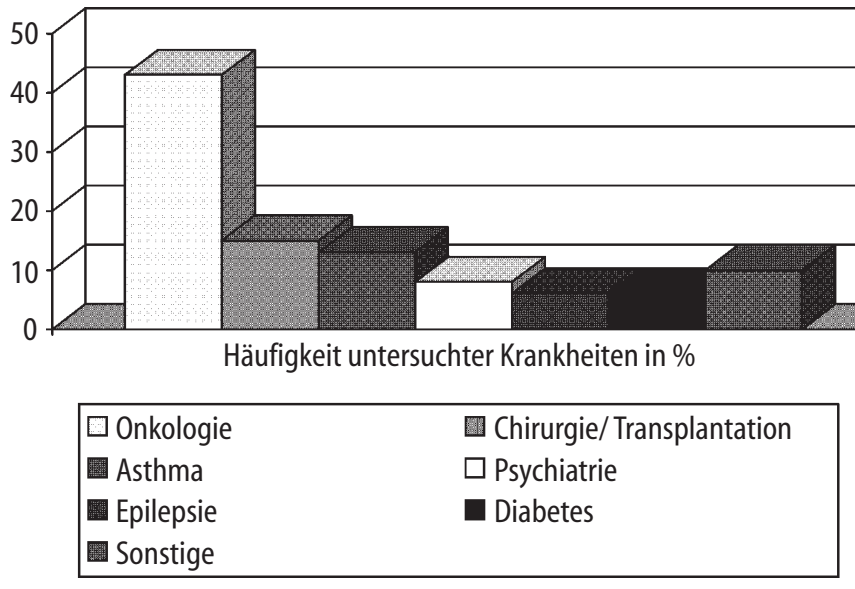

Abb. 2 Häufigkeiten der untersuchten Krankheiten (Publikationen Medline, 1994-1999)

nahmslos neueren Datums. Solche Daten sind jedoch wichtig im Rahmen der Prävention (Lebensqualitätsdaten als Hinweis auf Erkrankungsrisiken) und für Vergleichsmöglichkeiten mit chronisch kranken Kindern (z.B. Befragung repräsentativer Stichproben gesunder Kinder zum Thema Lebensqualität).

Die Altersverteilung der Stichproben in den Forschungsarbeiten hat ihren Schwerpunkt im Schul- und Pubertätsalter (Abb. 3). Studien, die sich auf die frühe Kindheit beziehen, sind selten. Auffällig ist, dass klare Altersgruppendifferenzierungen nur in wenigen Studien vorgenommen wurden und recht häufig Mischgruppen mit einer großen Altersspanne zu finden sind.

Die Literaturdurchsicht gibt Hinweise darauf, wie spärlich der Stand der Methodenentwicklung, der Stand der Anwendung und damit des Wissens über die Lebensqualität kranker Kinder ist. Epidemiologische Untersuchungen haben sich bisher kaum um die Beeinträchtigung der Lebensqualität von Kindern gekümmert [14], und auch im Bereich der öffentlichen Gesundheitsforschung gibt es nur wenige Arbeiten [15, $16,17,18]$. Bei über der Hälfte aller aufgefundenen Forschungsarbeiten handelt es sich um theoretische Arbeiten zum Thema Lebensqualität bei Kindern, das heißt sowohl konzeptionelle Arbeiten und Übersichtsarbeiten, als auch Arbeiten die sich mit der Entwicklung und Erprobung von Test- und Messverfahren beschäftigen (Abb.4).

\section{Spezifische Probleme der LQ-Erfassung bei Kindern}

Die Lebensqualitätsforschung bei Kindern muss sich mit spezifischen Fragen und Problemen auseinandersetzen, die, zumindest teilweise, als Ursache für die späte Entwicklung dieses Forschungsgebietes angesehen werden können.

Die Dimensionalität der Lebensqualität ist in Untersuchungen an Erwachsenen in einer Reihe qualitativer und quantitativer Studien sowohl im nationalen als auch im internationalen Bereich erhärtet worden $[19,20]$. Fraglich ist allerdings, inwieweit Kinder vergleichbare Dimensionen zur Beschreibung ihrer gesundheitsbezogenen Lebensqualität wählen würden. Literatur, die den Begriff „,Gesundheit” bei Kindern erörtert, legt nahe, dass das Konstrukt Gesundheit anders als das von Erwachsenen wahrgenommen wird [15]. Dies heißt, dass Bewertungen der ge- sundheitsbezogenen Lebensqualität von Kindern nicht von vornherein mit Studien zur Definition des Gesundheitsbegriffes aus Sicht der Erwachsenen gleichzusetzen sind. Dass Lebensqualität bei Kindern nicht durch Ableitung aus Konzepten verstanden werden kann, die für Erwachsene entwickelt wurden, beruht im wesentlichen auf den Umständen, dass Natur und Verlauf von Erkrankungen und emotionale und kognitive Reaktionen auf Gesundheit und Krankheit sich von denen Erwachsener unterscheiden, und dass Eltern bzw. Familie und Gleichaltrige im Kindesalter eine wesentliche Rolle spielen. Was bisher nur im Ansatz vorhanden ist, ist eine qualitative Forschung zur Frage, welche Dimensionen bzgl. des Befindens, des Erlebens und des Verhaltens Kinder aus ihrer eigenen Sicht haben.

Diese und andere wesentliche theoretisch-konzeptionelle Grundfragen zu Messung der gesundheitsbezogenen Lebensqualität bei Kindern und Jugendlichen wurden in der bestehenden Literatur bisher oft nur unzureichend beantwortet, wie zum Beispiel die Frage nach der Zuverlässigkeit kindlicher Urteilskraft, nach der Rolle und dem Zusammenhang von Selbst- und Fremdeinschätzung und nach der Reflexionsfähigkeit der Kinder in Abhängigkeit von Alter und Entwicklungsstand.

\section{Zuverlässigkeit kindlicher Urteilskraft}

Bei der Erfassung der gesundheitsbezogenen Lebensqualität von Erwachsenen sollten die Patienten, wenn möglich, immer selbst Auskunft über ihr Erleben und Verhalten geben. Die Frage nach der Zuverlässigkeit kindlicher Ur-

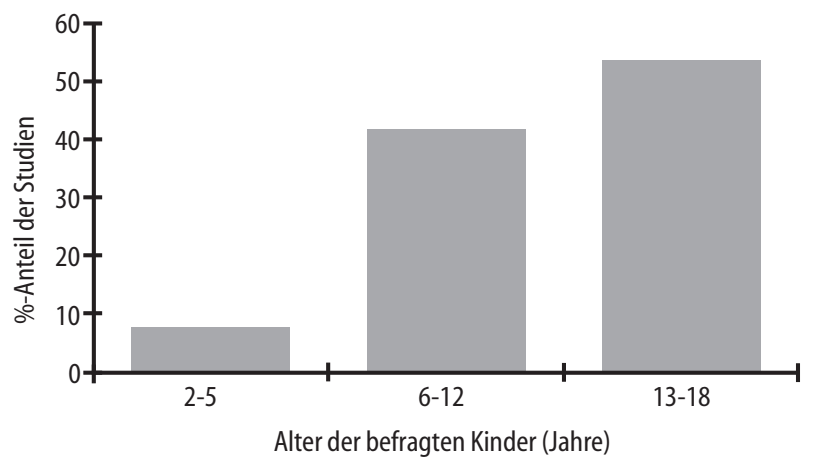

Abb. $3 \Delta$ Altersverteilung in Studien zu Lebensqualität bei Kindern und Jugendlichen 


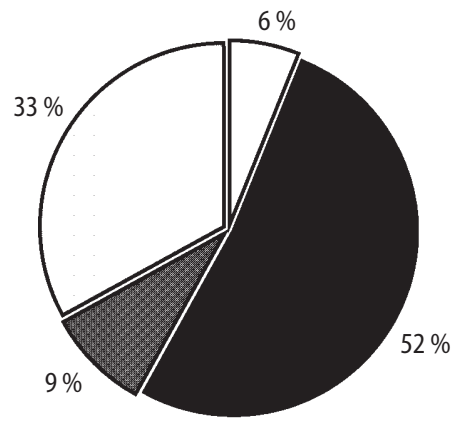

Allgemein
Theoretisch /
Konzeptionelle Arbeiten
Entwicklung und
Erprobung von
Meßverfahren
$\square$ Empirische Erhebungen
Krankheits-spezifisch

Abb. 4 Methodische Zugänge zur LQ-Forschung bei Kindern

(Publikationen Medline 1995-1999) teilskraft hat lange Zeit eine Lebensqualitätsforschung aus kindlicher Perspektive behindert. Obwohl die Einschätzung der Lebensqualität durch die Kinder selbst oftmals gefordert wird, findet das Prinzip patientengenerierter Datenerhebung erst in jüngerer Zeit Anwendung.

Gerade bei jüngeren Kindern sind Fremdbeurteilungsverfahren noch immer die Regel. Die Beurteilung erfolgt dabei entweder durch die Eltern, meistens durch die Mütter, als näherungsweise Antwortende (Proxys), oder durch Experten, in der Regel die behandelnden Ärzte. In mehr als 50\% aller identifizierten Studien zur gesundheitsbezogenen Lebensqualität von Kindern beurteilten Eltern das Wohlbefinden ihrer Kinder, in ca. 40\% wurden Pflegekräfte oder Ärzte mit dieser Aufgabe betraut. Nur in unter 10\% der Studien kamen Selbstbeurteilungsverfahren zum Einsatz (Abb. 5).

\section{„Die Lebensqualitätserfassung vor allem jüngerer Kinder erfolgt bisher meist über Fremdbeurteilungsverfahren, und deren Wert ist umstritten."}

Der Wert dieser Fremdeinschätzung ist äußerst umstritten. Einige Autoren betonen, dass Kinder und Erwachsene innerhalb verschiedener Bezugsrahmen funktionieren und sich dementsprechend in ihrem Verständnis von Lebensqualität und ihren Inhalten deutlich unterscheiden [9]. Während Eltern durchaus in der Lage sind verhaltensbezogene (externalisierte) Probleme, wie z.B. die Häufigkeit nächtlichen Aufwachens ihrer Kinder, zu beurteilen, fällt dies bei emotionsbezogenen (internalisierten) Problemen, wie z.B. Anspannung oder Traurigkeit, deutlich schwerer [21]. Ein Mangel an unmittelbarer Information führt zu einer eingeschränkten Kompetenz auf Seiten der Eltern beispielsweise in der Beurteilung von Erfahrungen der Kinder in der Schule oder in der Interaktion mit Freunden [20]. Guyatt et al. [22] stellten fest, dass Ärzte sich nicht auf die elterlichen Auskünfte über die gesundheitsbezogene Lebensqualität von Kindern verlassen können. Die elterlichen Angaben sind vielmehr eine zusätzliche Informationsquelle bezüglich der körperlichen Symptome, nicht aber eine Repräsentation des Krankheitserlebens und Fühlens der Kinder. Die Eltern kleinerer Kinder haben nur durch das beobachtbare Verhalten $\mathrm{Zu}$ gang zu dem kindlichen Erleben. Die Eltern älterer Kinder dagegen haben diesen Zugang auch über die verbalen kindlichen Berichte über das eigene Empfinden und Erleben. Dennoch können auch bei älteren Kindern (ca. ab zehn Jahre) Elternurteile nicht stellvertretend für Kinderurteile erhoben werden; wenn die Ärzte selbst die Kinder fragen, erhalten sie die notwendigen Informationen über das Verhalten und Erleben der Kinder unmittelbarer und zuverlässiger als von den Eltern.

Wenn geeignete, dem Alter entsprechende Methoden gefunden werden, können die Kinder auch recht früh in ihrem Leben zu ihrem Befinden befragt werden. Generell scheint es wichtiger, eine selbstberichtete Momentaufnahme der kindlichen Befindlichkeit zur Verfügung zu haben als nur eine, ebenfalls bias-anfällige, Beurteilung anderer Personen. Wo möglich, sollte die selbstbeurteilte Befindlichkeit der Kinder durch Angaben von anderen Beobachtern ergänzt werden, nicht aber mit dem Ziel einer Revision und skeptischen Betrachtung der Aussagen der Kinder, sondern als Ergänzung.

Neben der Möglichkeit, die gesundheitsbezogene Lebensqualität der Kinder stellvertretend über die Eltern zu ermitteln, spielt auch noch die Möglichkeit, die Lebensqualität der Eltern selbst in die Lebensqualitätsforschung mit einzubeziehen, eine besondere Rolle. Hierbei beurteilen die Eltern nicht als Proxies die gesundheitsbezogene Lebensqualität ihrer Kinder, sondern machen Angaben darüber, wie sie sich selbst, als „caregiver”, also als Versorgende der chronisch kranken Kinder, im Zusammenhang mit der Erkrankungssituation ihres Kindes fühlen, z.B. in sozialen Aktivitäten, dadurch dass ihnen die Versorgung ihres Kindes keine Zeit mehr lässt,

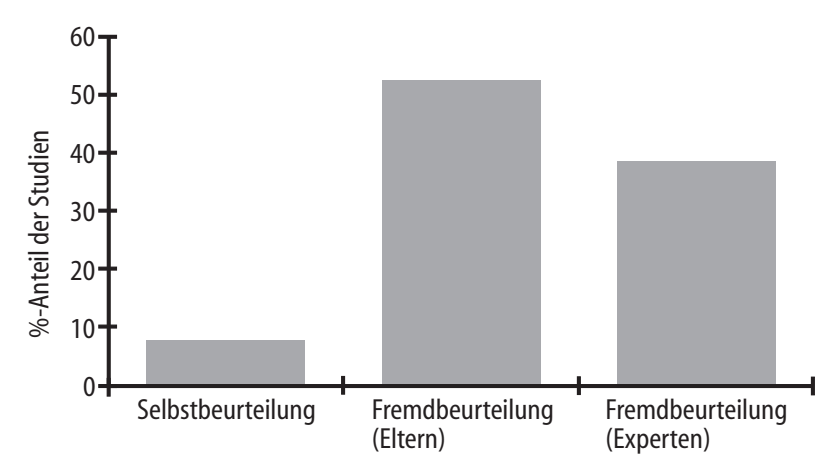

Abb. $5 \boldsymbol{\Delta}$ Verwendete Verfahren der Lebensqualitätsbeurteilung in Studien bei Kindern und Jugendlichen 
Kontakte mit Freunden aufrecht zu erhalten; oder in emotionaler Hinsicht dahingehend, dass sie vermehrt Ängste um die Gesundheit ihres kranken Kindes aufweisen. Die Information über die Belastung der Eltern durch die Erkrankung der Kinder und das Wohlbefinden der Eltern stellt einen wesentlichen Einflußfaktor für die Lebensqualität der Kinder dar [23].

\section{Alter und Entwicklungsstand}

Der oben angesprochene Aspekt der Selbstbeurteilung vs. Fremdbeurteilung wird durch andere Autoren [24] als Teil eines breiteren Problems gesehen, nämlich der Angemessenheit der Erfassung an Alter und Entwicklungsstand des Kindes. Die unterschiedlichen Antworten zwischen Kindern und Eltern oder medizinischem Personal sind stark von der kognitiven Entwicklung des Kindes abhängig. Der kognitive Bereich umfasst das Wissen der Kinder über die Krankheit und das Konzept, das Kinder sich von ihrer Krankheit machen. Die kindlichen Überzeugungen, mit den Anforderungen und Belastungen selbst eigenverantwortlich fertig werden zu können, haben einen entscheidenden Einfluß auf die Lebensqualität [25]. Dass die eigene Wahrnehmung und die Wahrnehmung eigener Emotionen ein entwicklungsbedingter Faktor ist, ist in der Literatur gut dokumentiert [26]. Kinder unter zehn Jahren sind z.B. weniger in der Lage, komplexe Situationen oder Emotionen $\mathrm{zu}$ verstehen und zu beurteilen als ältere Kinder. Pantell und Lewis [27] definieren als integralen Bestandteil des $\mathrm{Ge}$ sundheitszustands von Kindern die Fähigkeit, altersentsprechende Tätigkeiten auszuführen, was körperliche, emotionale und soziale Aktivität erfordert. Solche funktionalen Aspekte von Gesundheit sind besonders in epidemiologischen Studien betont worden [28].

Die Frage nach den Änderungen in den kindlichen Entwicklungsphasen ist in der Pädiatrie vor allem dann von Bedeutung, wenn z.B. die Anwendung von Lebensqualitätsfragebögen $\mathrm{zu}$ unterschiedlichen Messzeitpunkten bei longitudinalen Studien erfolgt. Hierbei können sich von Messzeitpunkt zu Messzeitpunkt Unterschiede in der Beurteilung der Lebensqualität ergeben, die nicht auf eine bestimmte Behandlung, sondern auf die fortschreitende

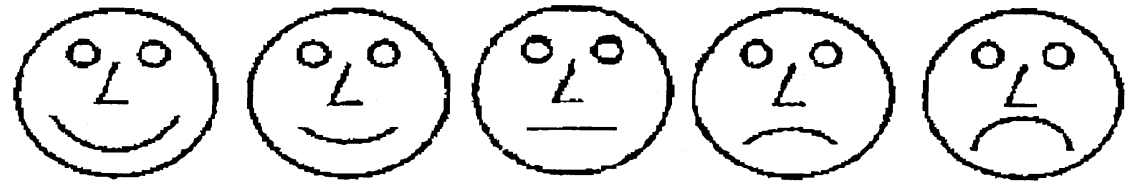

Abb.6 $\Delta$ Beispiele für Gesichtsantwortskalen aus dem Childhood-Asthma-Questionaire [Christie et al. 1991]

kognitive Entwicklung der Kinder zurückzuführen sind. Oftmals können gerade kleine Kinder unter sechs bis acht Jahren die Bedeutung einiger Fragen der als „paper-pencil-form” und allgemein für Kinder konzipierten Lebensqualitätsbögen nicht vollständig verstehen und beantworten, da ihre Lese- und Abstraktionsfähigkeiten nicht ausreichen.

Einige Autoren kommen daher der Forderung nach Berücksichtigung der kindlichen Entwicklungsfortschritte mit unterschiedlichen Versionen eines Fragebogens für unterschiedliche Altersgruppen nach. So haben z.B. Christie et al. [29] einen asthmaspezifischen Fragebogen zur Abschätzung des Therapieerfolgs bei Kindern mit chronisch obstruktiven Atemwegserkrankungen für drei unterschiedliche Altersgruppen (vier bis sieben, acht bis elf und 12 bis 14 Jahre) entwickelt. Die likert-skalierten Antwortkategorien in der Version für die älteren Kinder wurde in der Version für die jüngeren Kinder durch Gesichterskalen (happy and sad faces) (Abb.6) ersetzt, um den Schwierigkeitsgrad bei der Beantwortung zu verringern.

\section{Geschlechtszugehörigkeit}

Ebenso wie die Entwicklungsangemessenheit von Messverfahren ist auch die Frage nach der Unterschiedlichkeit der Geschlechtszugehörigkeit bei der Erfassung der Lebensqualität von Kindern von zentraler Bedeutung. Während der Pubertät spielen Konzepte z.B. wie Selbstwertgefühl und körperliches Selbstbild eine besonders wichtige und bei Mädchen und Jungen unterschiedliche Rolle. Bei Mädchen lassen sich gerade in Bezug auf das Körperbild mehr Probleme als bei Jungen feststellen [30]. Die bis zu diesem Zeitpunkt durchgeführten Studien mit chronisch kranken Jugendlichen zeigen, dass das Geschlecht eine zu berücksichtigende Variable ist, wenn die gesundheitsbezogene Lebensqualität von Jugendli- chen in ihrer ganzen Komplexität erfasst werden soll.

\section{"Das Geschlecht ist eine zu berücksichtigende Variable, wenn die gesundheits- bezogene Lebensqualität von Jugendlichen in ihrer ganzen Komplexität erfasst werden soll."}

Zum Beispiel weisen weibliche Teenager mit insulinabhängigem Diabetes mehr depressive Symptome als männliche Teenager auf. LaGreca et al. [31] und Austin et al. [32] konnten feststellen, dass epilepsiekranke Mädchen mehr Angst und weniger Zufriedenheit, aber auch eine positivere Einstellung gegenüber der Krankheit und ein besseres Beziehungsnetz als Jungen zeigen. Auch bei asthmakranken Mädchen ließen sich mehr Angst, weniger Zufriedenheit und ein geringeres Selbstbewußtsein als bei Jungen erkennen.

Schritt für Schritt findet in der Lebensqualitäts-Forschung bei Kindern die Notwendigkeit der Entwicklung kindgerechter Verfahren, die die altersentsprechende kognitive Leistungsfähigkeit der Kinder einbeziehen, zunehmende Beachtung [33]. Die Einbeziehung der Entwicklungspsychologie und die weitere Erforschung der Krankheitsund Gesundheitskonzepte der Kinder waren hierfür notwendige Voraussetzungen. Die früher häufig geübte Praxis, ursprünglich für Erwachsene vorgesehene Instrumente kindgerecht zu modifizieren, gilt heute als nicht mehr sinnvoll [34], zumal inzwischen, gerade im angloamerikanischen Sprachraum, Instrumente vorliegen, die spezifisch für Kinder konzipiert und validiert wurden [35]. 
Tabelle 1

Krankheitsübergreifende Instrumente zur Erfassung der Lebensqualität von Kindern und Jugendlichen

\begin{tabular}{|c|c|c|c|c|c|c|}
\hline Nr. & Verfahren (Autoren) & Ursprungsland & Altersgruppe & Kennzeichen & Reliabilität & Validität \\
\hline 1 & $\begin{array}{l}\text { QWB Quality of Well-being } \\
\text { Scale (Kaplan et al. 1981) }\end{array}$ & USA & Kleinkinder und Kinder & $\begin{array}{l}18 \text { Items } 4 \text { Skalen } \\
\text { Experten-/Elternbeurteilung }\end{array}$ & 0.90 & Kriterium-, konvergente $\mathrm{V}$. \\
\hline 2 & $\begin{array}{l}\text { Ontario Child Health Study } \\
\text { (Boyle et al. 1988) }\end{array}$ & Kanada & $4-16$ & $\begin{array}{l}5 \text { Skalen Selbst-, Eltern-, } \\
\text { Lehrerbeurteilung }\end{array}$ & $0.70-0.90$ & $\begin{array}{l}\text { Konstrukt-, konvergente/ } \\
\text { diskriminante V. }\end{array}$ \\
\hline 3 & $\begin{array}{l}\text { BFW/J Berner Fragebogen } \\
\text { zum Wohlbefinden (Jugend- } \\
\text { liche) (Grob et al. 1989) }\end{array}$ & Schweiz & $14-20$ & $\begin{array}{l}39 \text { Items } 6 \text { Skalen, } \\
\text { Selbstbeurteilung }\end{array}$ & $0.52-0.78$ & Konstrukt-, diskriminante V. \\
\hline 4 & $\begin{array}{l}\text { CHRIs Children's Health } \\
\text { Rating Skalen (Maylath 1990) }\end{array}$ & USA & $10-13$ & $\begin{array}{l}17 \text { Items } 5 \text { Skalen } \\
\text { Selbstbeurteilung }\end{array}$ & 0.83 & Kriteriums-V. \\
\hline 5 & $\begin{array}{l}\text { CHQ Child Health } \\
\text { Questionnaire } \\
\text { (Landgraf et al. 1993) }\end{array}$ & USA* & $5-18$ & $\begin{array}{l}28,50,69 \text { oder } 98 \text { Items } 9 \text { Skalen } \\
\text { Selbst-/Elternbeurteilung }\end{array}$ & $0.70-0.98$ & Kriterium-, diskriminante $\mathrm{V}$. \\
\hline 6 & $\begin{array}{l}\text { CHIP Child Health and Illness } \\
\text { Profile (Starfield et al. 1993) }\end{array}$ & USA* & $11-17$ & $\begin{array}{l}275 \text { Items } 6 \text { Skalen } \\
\text { Selbstbeurteilung }\end{array}$ & $>0.70$ & $\begin{array}{l}\text { konvergente/ diskrimin- } \\
\text { nante } \mathrm{V} \text {. }\end{array}$ \\
\hline 7 & $\begin{array}{l}\text { CQOL Child Health Related } \\
\text { Quality of Life (Graham } \\
\text { et al.1994) }\end{array}$ & GB & $9-15$ & $\begin{array}{l}46 \text { Items Selbst- } / \\
\text { Elternbeurteilung }\end{array}$ & $>0.70$ & $\begin{array}{l}\text { Konstrukt-, diskrimi- } \\
\text { nante V. }\end{array}$ \\
\hline 10 & $\begin{array}{l}\text { GCQ Generic Child Quality of } \\
\text { Life Measure (Collier et al. 1995) }\end{array}$ & USA & $6-16$ & 25 Items Selbstbeurteilung & $0.69-0.78$ & Konstrukt-V. \\
\hline 12 & TACQOL (Verrips et al. 1997) & Niederlande* & $6-15$ & $\begin{array}{l}56 \text { Items } 7 \text { Skalen Selbst-/ } \\
\text { Elternbeurteilung }\end{array}$ & $0.71-0.89$ & Konstrukt-, diskriminante V. \\
\hline 13 & $\begin{array}{l}\text { HAY How are you? } \\
\text { (Bruil et al. 1997) }\end{array}$ & Niederlande* & $8-13$ & $\begin{array}{l}5 \text { Skalen + Module Selbst-/ } \\
\text { Elternbeurteilung }\end{array}$ & $0.71-0.83$ & Konstrukt-, konvergente V. \\
\hline 14 & $\begin{array}{l}\text { DUC-25 (Koopman } \\
\text { et al. 1998) }\end{array}$ & Niederlande & $6-16$ & $\begin{array}{l}25 \text { Items } 4 \text { Skalen } \\
\text { Selbstbeurteilung }\end{array}$ & $0.75-0.76$ & Konstrukt-, diskriminante V. \\
\hline 15 & $\begin{array}{l}\text { KINDL (Bullinger et al. 1994) } \\
\text { (modifiziert: Ravens-Sieberer } \\
\text { \& Bullinger 1998) }\end{array}$ & Deutschland & $4-78-1213-16$ & $\begin{array}{l}24 \text { Items } 6 \text { Skalen + Module } \\
\text { Selbst-/Elternbeurteilung }\end{array}$ & $0.70-0.95$ & $\begin{array}{l}\text { Konstrukt- diskriminante/ } \\
\text { konvergente V. }\end{array}$ \\
\hline 16 & $\begin{array}{l}\text { VSP-A perceived health of } \\
\text { adolescent (Siméoni et al. } \\
\text { 1998) }\end{array}$ & Frankreich* & $11-17$ & $\begin{array}{l}54 \text { Items } 7 \text { Skalen Selbst-/ } \\
\text { Elternbeurteilung }\end{array}$ & $0.81-0.87$ & Konstrukt-V. \\
\hline 17 & $\begin{array}{l}\text { AUQUEl (Manificat \& Dazord } \\
\text { 1998) }\end{array}$ & Frankreich* & $4-12$ & $\begin{array}{l}27 \text { Items Selbstbeurteilung } \\
\text { (Elternbeurteilung in Ent- } \\
\text { wicklung) }\end{array}$ & $\begin{array}{l}\text { keine } \\
\text { Angaben }\end{array}$ & keine Angaben \\
\hline 18 & $\begin{array}{l}\text { PedsQL Pediatric Quality of } \\
\text { Life Inventory (Varni et al. } \\
\text { 1999) }\end{array}$ & USA & $2-18$ & $\begin{array}{l}15 \text { Items } 11 \text { Skalen + Module } \\
\text { Selbst-/ Elternbeurteilung }\end{array}$ & $0.83-0.86$ & Konstrukt-V. \\
\hline
\end{tabular}

*dt. Übersetzung vorhanden

\section{Messinstrumente zur Erfassung der gesundheitsbezogenen Lebensqualität}

Bezieht man sich bei der Erfassung der gesundheitsbezogenen Lebensqualität auf Messinstrumente wie Fragebögen oder Interviews, so zeigt sich, dass zum Thema Lebensqualität bei Kindern in der Literatur mittlerweile eine Reihe von Messinstrumenten existiert. Zu unterscheiden ist zwischen Instrumenten, die sich eher auf die Funktionsfähigkeit der
Kinder beziehen, Instrumenten, die eine Beurteilung der Befindlichkeit ermöglichen und Messverfahren, die die gesundheitsbezogene Lebensqualität von Kindern und Jugendlichen multidimensional erfassen. Natürlich gibt es in der internationalen Literatur eine Reihe von Arbeiten über Aspekte des Befindens und Verhaltens von Kindern, die auch den Lebensqualitätsdimensionen zuzurechnen sind. Allerdings sind diese Verfahren häufig unidimensional, d.h. sie erfassen nur einen bestimmten Aspekt aus dem Lebensqualitätskonzept, z.B. die Funktionsfähigkeit unter Vernachlässigung der Befindlichkeit oder umgekehrt.

\section{Erfassung der Funktionsfähigkeit}

Amerikanische Forscher in der pädiatrischen Rehabilitation haben die Bedeutung sogenannter ,functional outcome measures" betont [36]. In einem Überblick über die bisherige Forschung zu Funktionsindikatoren bei Kindern stel- 
len Haley et al. [37] den konzeptuellen Rahmen und Meßinstrumente für den anglo-amerikanischen Raum dar. Grundlegend für die Erfassung von Funktionsfähigkeit bei Kindern ist die Differenzierung entsprechend der WHO-Einteilung nach ,impairment, disability und handicap”, wobei mit den sogenannten ,impairments" vor allem die Ausführung von motorischen Aktivitäten gemeint ist, mit „disability” die Einschränkung in bezug auf Mobilität und Selbstversorgung und mit „handicap” Probleme bei der Erfüllung sozialer Rollen in der Schule und im Sozialbereich. Funktionen, die im Bereich z.B. der Selbstversorgung gemessen werden, beziehen sich aufs Essen, die Körperpflege, aufs Anziehen und im Bereich der sozialen Funktionen z.B. auf Kommunikation, soziale Interaktion und tägliche Routinen. Der Schwerpunkt liegt hier auf der Fertigkeit, d.h. der Fähigkeit, bestimmte Aktivitäten auszuführen (Skills-Ansatz).

Im Gegensatz zur Entwicklungspsychologie, in der verschiedene Inventare zur Feststellung von Entwicklungsstufen entwickelt wurden [38], bezieht sich die Erfassung von Funktionsfähigkeit im Kindesalter speziell auf die Probleme, die die meist erkrankten Kinder haben, d.h. sie sind auch stets standardisiert für funktionsbeeinträchtigte Kinder und legen nicht so sehr auf Normalität (Referenz zu gesunden Kindern) als auf Unabhängigkeit im täglichen Leben wert (optimaler Funktionszustand vor dem Hintergrund einer spezifischen Erkrankung). Wie auch andere Messinstrumente können „functional outcome measures" prädiktive, diskriminative und evaluative Funkionen haben.

\section{Erfassung der Befindlichkeit}

Während die Messung von Funktionsfähigkeit als einem Bereich der Lebensqualität von Kindern besonders in der amerikanischen Rehabilitationsforschung verfolgt wurde, stellt die Erfassung der Befindlichkeit von Kindern im klinischen Bereich ein etabliertes Forschungsfeld dar [39]. Unter Befindlichkeit werden hier Stimmungsdimensionen wie Reizbarkeit, Ängstlichkeit und Depressivität verstanden, die sowohl im Rahmen psychiatrischer Erkrankungen als auch im Rahmen von psychosomatischen Erkrankungen von Bedeutung sind. Obwohl eine große Vielzahl von In- strumenten zur Erfassung der Befindlichkeit existiert, gibt es dennoch nur wenige, speziell für Kinder entwickelte Befindlichkeitsskalen, die in der Lebensqualitätsforschung eingesetzt werden. Dazu gehören im deutschen Sprachraum vor allem Selbst- und Fremdbeurteilungssysteme aus dem Bereich der Kinder- und Jugendpsychiatrie, die auf die Entdeckung von psychiatrischer Morbidität orientiert sind, was eine Anwendung der Verfahren bei somatischen Erkrankungen erschwert. Gemeinsam ist diesen Verfahren, dass sie die Dimension der Befindlichkeit entweder hinsichtlich psychiatrischer Morbidität oder Stimmungsschwankungen operationalisieren, wobei hier nur Teilkomponenten der Lebensqualität, nämlich die psychische und die physische angesprochen werden. Im Unterschied dazu versuchen Messinstrumente zur Erfassung der gesundheitsbezogenen Lebensqualität und des subjektiven Gesundheitszustands von Kindern und Jugendlichen entsprechend des multidimensionalen Konstrukts mehrere verschiedene Komponenten gleichzeitig zu erfassen.

\section{Erfassung der Lebensqualität}

Bei den Verfahren zur multidimensionalen Erfassung der gesundheitsbezogenen Lebensqualität ist zu unterscheiden zwischen Ansätzen, die die Lebensqualität krankheitsübergreifend (generisch), d.h. unabhängig vom aktuellen Gesundheitszustand der Kinder untersuchen, und solchen, die spezifisch bestimmte Erkrankungen thematisieren. Krankheitsübergreifende Verfahren sind besonders dann einzusetzen, wenn Daten zur Verteilung von Lebensqualitätsbeurteilungen aus epidemiologischen Studien zugrundegelegt oder Screeninguntersuchungen durchgeführt werden sollen, wohingegen krankheitsspezifische Verfahren zur Evaluation von verschiedenen Behandlungsalternativen geeignet sind. Zur Übersicht über die zunehmende Anzahl krankheitsspezifischer Instrumente für Kinder und Jugendliche kann auf vielfältige Übersichtsarbeiten verwiesen werden [40, 41, 42]. Nachfolgend werden die international und national verfügbaren generischen Instrumente zur Erfassung der gesundheitsbezogenen Lebensqualität bei Kindern und Jugendlichen tabellarisch (Tabelle 1) (geordnet nach Publikations- jahr) dargestellt und die in der deutschen Sprache verfügbaren und in Deutschland am häufigsten eingesetzten Verfahren kurz beschrieben.

\section{„Child Health Questionnaire" (CHQ)}

Ein Beispiel für ein multidimensionales und in der Durchführung flexibel angelegtes krankheitsübergreifendes Verfahren ist der "Child Health Questionnaire” [43], der, ähnlich wie der SF-36, aus der amerikanischen Medical Outcome Study (MOS) entwickelt wurde. Der „Child Health Questionnaire” besteht aus einer allgemeinen Gesundheitseinschätzung auf einer Skala zwischen „,ausgezeichnet” und ,schlecht” und bezieht sich auf körperliche Tätigkeiten des Kindes, alltägliche Beschäftigungen des Kindes, Schularbeiten, Schmerzen, Beschwerden, Zurechtkommen mit anderen, allgemeines Wohlbefinden, Zufriedenheit mit verschiedenen Lebensbereichen und Einschätzung des Gesundheitszustands, wobei diese Einschätzung des Gesundheitszustands sowohl von den Müttern als auch von den Kindern selbst vorgenommen werden kann. Die Mutter kann zusätzlich noch eine Einschätzung ihrer eigenen allgemeinen Gesundheit und der Wirkung ihres Kindes auf sie hinsichtlich seiner Befindlichkeit und seines Gesundheitszustandes durchführen. Das Verfahren liegt als Fremdbeurteilungsverfahren (50 Items, Kurzform 28 Items) und als Selbstbeurteilungsverfahren (87 Items) vor und ermöglicht somit seinen Einsatz in einer großen Bandbreite von Studien. Bisherige psychometrische Analysen im amerikanischen Sprachraum zeigen, dass das Messinstrument in der Lage ist, bei guter Reliabilität verschiedene Krankheitsgruppen $\mathrm{zu}$ unterscheiden. Für den deutschen Sprachraum wurde der „Child Health Questionnaire" nach einem detaillierten Vorwärts-/Rückwärtsprozedere übersetzt und in einer Untersuchung an 350 gesunden Kindern aus einer umweltpsychologischen Studie psychometrisch geprüft [44]. Die vorliegenden Daten zeigen, dass auch der deutsche „Child Health Questionnaire" gute bis befriedigende Eigenschaften hat und dass sein Einsatz in epidemiologischen und klinischen Studien zu rechtfertigen ist; die lange Ausfüllzeit des Bogens (ca. 35 Minuten) stellt jedoch oftmals ein Problem 
bei der Anwendung des „Child Health Questionnaires" in Studien dar.

\section{„TACQOL-Fragebogen”}

Der in den Niederlanden entwickelte „TACQOL-Fragebogen” - TNOAZL Children's Quality of Life [45] - wurde als generisches Instrument besonders für den Einsatz in der medizinischen Forschung und für klinische Studien konzipiert und liegt in zwei Formen (Kinder- und Elternform) vor. Der Fragebogen wurde an acht- bis elfjährigen Kindern und ihren Eltern in den Niederlanden an einer gesunden Stichprobe normiert und besteht aus 56 Items, die in sieben Dimensionen mit acht Items pro Skala zusammengefasst werden. Die Skalen sind physische Beschwerden, Bewegungsfähigkeit, Selbständigkeit, kognitive Funktion, soziale Funktion, positive Emotionen und negative Emotionen. Die Autoren betrachten gesundheitsbezogene Lebensqualität als eine Kombination von Gesundheitszustand und der emotionalen Reaktion auf diesen Gesundheitszustand. Die Fragen sind so aufgebaut, dass sie mit einer Antwortkategorie, die in drei Stufen von nie - manchmal - oft die Quantität des Vorliegens einer Einschränkung und, falls diese Einschränkung besteht, die emotionale Reaktion auf diese Einschränkung mit einer vierstufigen Skala erfasst, die die eigene Befindlichkeit von sehr gut - nicht so gut - eher schlecht schlecht abbildet. Die interne Konsistenz der Skalen (Cronbach's Alpha) reicht von 65 bis 84 für die gesundheitsbezogene Lebensqualität und 64 bis 84 für den Gesundheitszustand. Für die Gesamtskala konnte mit der Multi-Trait-MultiMethod-Analyse und dem structural equation modelling ein guter Skalenfit nachgewiesen werden. Die Stärken der Skala bestehen darin, dass sie den Gesundheitsstatus erfassen, gute Reliabilität und Validität aufzeigen, dass die krankheitsbezogenen Fragen als Fragen nach Emotionen abgebildet werden und dass eine Elternform erhältlich ist. Schwächen des Instruments bestehen darin, dass sie nur für ein geringes $\mathrm{Al}$ tersspektrum zur Verfügung steht, und dass die Fragen ursprünglich nicht mit Kindern entwickelt wurden. Chronisch kranke Kinder wurden nicht in die Normierung der Skala miteinbezogen. Der Fragebogen ist, neben anderen, auch in den Sprachen Englisch und Deutsch vorhanden, allerdings in diesen noch nicht psychometrisch geprüft.

\section{„How are you?-Fragebogen” (HAY)}

Der „How are you?-Fragebogen” (HAY), [46], ein ebenfalls in den Niederlanden entwickeltes Instrument, misst sowohl krankheitsübergreifende als auch krankheitsspezifische Aspekte des subjektiven Wohlbefindens mit 22 Items. Der krankheitsübergreifende Teil umfasst fünf Dimensionen (physische Funktionsfähigkeit, kognitive Funktionsfähigkeit, soziale Funktionsfähigkeit, physische Beschwerden und positive emotionale Zustände), der krankheitsspezifische Teil bildet vier Dimensionen ab (Symptome in Bezug auf die Erkrankung, Selbstmanagement, Selbstkonzept, der Krankheit zugeordnete Emotionen). Die Antwortkategorien werden für die meisten Fragen mit smiley-faces oder auch Piktogrammen abgebildet. Neben dem Fragebogen für Kinder gibt es ein zusätzlich äquivalentes Instrument für Eltern. Die psychometrische Prüfung des Fragebogens erfolgte an insgesamt 569 Kindern mit chronischen Erkrankungen und 344 gesunden Kindern im mittleren Alter von 10,3 Jahren und ihren Eltern. Die Reliabilität der berichteten Skalen (Cronbach's Alpha) reicht von 0.71 bis 0.86 . Im Hinblick auf die konvergente Validität des Instrumentes konnten Korrelationen mit der Child Behaviour Checklist für verschiedene Dimensionen berechnet werden, die von 0.2 bis 0.59 reichen, wobei die Korrelationen als signifikant berichtet wurden $(p<=0,001)$. Die Stärke des Instruments besteht darin, dass es sowohl generische als auch krankheitsspezifische Aspekte der Lebensqualität beinhaltet und qualitative Bereiche abgefragt werden. Es ist möglich, den Fragebogen in einem breiten Altersspektrum einzusetzen, wobei insgesamt gute psychometrische Qualitäten berichtet werden. Der Fragebogen ist, neben niederländisch, auch in den Sprachen Englisch und Deutsch vorhanden, allerdings in diesen noch nicht psychometrisch geprüft.

\section{„Health Related Quality of Life” (VSP-A)}

Das französische Instrument „Health Related Quality of Life" (VSP-A), [47], ein ebenfalls multidimensionales Instrument, wurde entwickelt, indem die Items durch Interviews mit Jugendlichen gewonnen wurden. Die Itemgenerierung erfolgte in Zusammenarbeit mit Jugendlichen, Eltern und Lehrern. Der Fragebogen wurde in einer großen Public-Health-Studie an ca. 3000 Jugendlichen im Alter von 11 bis 16 Jahren und ihren Eltern eingesetzt. Der Fragebogen besteht aus 36 Items und hat fünf Likertskalierte Antwortkategorien. Die Antwortkategorien des Fragebogens reichen von nie bis immer. Insgesamt werden acht Dimensionen abgebildet (negative psychologische Gesundheit, Beziehung zu Eltern, Vitalität-Energie, Beziehung zu Freunden, Freizeitaktivitäten, Schule, Sexualleben, Inaction/idleness). Die interne Konsistenz der Skalen weist ein Cronbach's Alpha von über 75 auf. Die Test-Retest-Reliabilität liegt für die meisten Skalen bei um die 70. Im Hinblick auf die konvergente Validität reichen Korrelationen mit der Global Health Perception scale von 11 bis 50 (alle signifikant). Die Stärken des Instruments liegen darin, dass die Fragen zusammen mit Jugendlichen entwickelt wurden und hier besonders auch Fragen zu freundschaftlichen Beziehungen und Sexualleben der Jugendlichen enthalten sind. Ebenfalls ist eine Elternform erhältlich. Eine Schwäche des Fragebogens könnte darin gesehen werden, dass er nur für ein sehr schmales Altersspektrum entwickelt wurde und kleinere Kinder nicht damit befragt werden können sowie die Tatsache, dass die Skala inaction/idleness insgesamt schlechte psychometrische Gütekriterien aufweist. Die Standardisierung des Fragebogens erfolgte nicht an einer repräsentativen Stichprobe der Allgemeinbevölkerung, und chronisch kranke Kinder wurden nicht einbezogen. Qualitative Aspekte von Lebensqualität können mit dem Fragebogen erfasst werden. Eine deutsche Übersetzung und Validierung des Instruments findet derzeit am Institut für medizinische Psychologie der Universität Hamburg statt.

\section{"AUQUEI"}

Der zweite französische Fragebogen „AUQUEI” [48] wurde ebenfalls in Frankreich zusammen mit Kindern entwickelt, deren Alter von etwas über drei Jahren bis zwölf Jahren reichte. Von die- 
sen Kindern waren ungefähr 60\% gesund; die anderen Kinder hatten chronische Erkrankungen, hauptsächlich HIV oder Niereninsuffizienz. Der Fragebogen wurde entwickelt, um Aussagen zur Lebensqualität speziell kleinerer Kinder qualitativ zu erfassen. Ein kleines Buch mit Zeichnungen enthält 27 Items, die insgesamt verschiedene Bereiche kindlicher Lebensqualität abdecken (Familienleben, Sozialleben, Aktivitäten von Kindern (Schule und Freizeit) Gesundheit). Jede Frage oder Zeichnung deckt die kindliche Zufriedenheit oder Emotion in einer Situation, z.B. in der Schule, ab. Die Antwortkategorie besteht aus Zeichnungen, die Kinder in vier verschiedenen emotionalen Zuständen zeigen und reichen von sehr unzufrieden bis sehr zufrieden. Die Kinder werden gebeten, zu jeder Frage ein ähnliches Szenario bezogen auf sich selber auszumalen, in dem sie sehr glücklich, glücklich, nicht so glücklich oder sehr unglücklich sind. Für den Fragebogen sind psychometrische Gütekriterien wie Reliabilität oder Validität nicht berichtet; berichtet wird allerdings, dass das Instrument zwischen gesunden und kranken Kindern differenzieren kann. Parallel zur Befragung der Kinder können die Eltern eine entsprechende Version ausfüllen. Die Stärken des Instruments bestehen sicherlich darin, dass auch kleinere Kinder befragt werden können, und dass es hier möglich ist, Zufriedenheit und Emotionen qualitativ zu messen, sowie darin, dass gesundheitsbezogene Fragen angesprochen werden. Die Schwächen des Instruments liegen darin, dass psychometrische Gütekriterien nicht berichtet werden, und dass nur über eine kleine Stichprobe Informationen vorliegen.

\section{,I,Child Health and IIIness Profile - Adolescent Edition" (CHIP-AE)}

Das Health and Ilness Profile für Jugendliche [49], ursprünglich in den USA entwickelt, erfasst in erster Linie funktionale Aspekte der Lebensqualität. Der Fragebogen, konzipiert für Jugendliche von elf bis 17 Jahren, ist in sechs Dimensionen der Gesundheit organisiert („Unzufriedenheit”, „Zufriedenheit mit der Gesundheit”, „Beschwerden”, „Erreichen von sozialen Zielen”, „Risikoverhalten"). Die 184 Items wurden von Experten auf der Grundlage bestehender
Literatur generiert und lassen sich in 20 Subdimensionen gliedern, die dann wiederum die sechs genannten Dimensionen bilden. Für den Fragebogen ist eine Auswertung in Form eines Profils der Gesundheit und der Lebensqualität möglich.

Das Instrument wurde insgesamt an 877 gesunden Jugendlichen in Schulen getestet und zusätzlich an einer Gruppe von 133 chronisch kranken Kindern mit unterschiedlichen Diagnosebilder, u.a. zystische Fibrose, Rheuma und Infektionskrankheiten. Hinsichtlich der psychometrischen Gütekriterien wird für den Bogen eine interne Konsistenz (Cronbach's Alpha) für alle Subdimensionen höher als 70 angegeben. Die Test-/Retest-Reliabilität wird als angemessen beschrieben; ebenfalls berichtet wird, dass die Korrelationen zwischen Subdimensionen und anderen Lebensqualitätsinstrumenten hoch sind, so liegt z.B. die Korrelation zwischen der Subdimension ,Selbstbewusstsein” und Beurteilungen aus dem Children Depression Inventory über 40. Für den Fragebogen ist eine Elternversion verfügbar, wesentliche Unterschiede zwischen Eltern- und Kinderurteilen werden nicht angegeben.

Die Stärken des Instruments liegen in der psychometrischen Güte und der Möglichkeit, Gesundheitsprofile zu entwickeln. Innerhalb des Bogens können auch qualitative Aspekte der Gesundheit in Form von Zufriedenheit mit derselben erfasst werden, in dem Instrument sind gesundheitsbezogene Fragen integriert, ebenso wie Fragen zum Umgang mit der Krankheit (Coping). Schwächen des Instruments liegen sicherlich darin, dass es nur für Jugendliche angewendet werden kann und so nur einen schmales Altersspektrum abdeckt, und das die Stichprobe, an der der Fragebogen standardisiert wurde, nicht repräsentativ und auch nicht besonders groß ist. Der Fragebogen ist insgesamt sehr lang und die Items wurden nicht mit Kindern zusammen generiert.

\section{„Berner Fragebogen zum Wohlbefinden Jugendlicher" (BFW)}

Der Berner Fragebogen zum Wohlbefinden Jugendlicher dient (BFW) [50] der Erfassung von Zufriedenheit und negativer Befindlichkeit. Der Fragebogen, der bei Kindern und Jugendlichen im Alter um 14 Jahren einzusetzen ist, beinhaltet 39 Items (die Bearbeitungszeit beträgt zehn Minuten) die nach Faktorenanalyse sechs Dimensionen des Wohlbefindens messen, nämlich: positive Lebenseinstellung, Problembewusstsein, körperliche Beschwerden und Reaktionen, Selbstwert, depressive Stimmung und Lebensfreude. Der Fragebogen wurde sorgfältig konstruiert und umfassend an großen Stichproben von ca. 6000 repräsentativen Schweizer Jugendlichen und 3844 Jugendlichen in sieben westlichen (einschließlich Deutschland) und östlichen Ländern psychometrisch geprüft. Re-Test-Reliabilitätskoeffizienten werden als befriedigend bezeichnet, konvergent fanden sich Zusammenhänge zwischen Wohlbefinden und der Offenheitsskala des MMPI sowie einigen Dimensionen des Freiburger Persönlichkeitsinventars FPI. Als Zeichen der Konstruktvalidität konnte eine Korrelation mit vergleichbaren und anderen Selbsteinschätzungsskalen festgestellt werden. Als Hinweis für die diskriminante Validität des Instruments konnte zwischen klinisch depressiven und nichtklinisch depressiven Jugendlichen unterschieden werden.

\section{„Fragebogen zur Erfassung der gesundheitsbezogenen Lebensqualität von Kindern und Jugendlichen" (KINDL)}

Während die meisten Lebensqualitätsinstrumente für Kinder in englischer Sprache entwickelt und in einem weiteren, methodologisch aufwendigen Schritt übersetzt wurden, liegt mit dem krankheitsübergreifenden KINDL-Fragebogen ein deutschsprachiges Instrument vor (ursprünglich entwickelt von Bullinger et al. 1994, revidiert von Ravens-Sieberer und Bullinger 1998) [51,52], das bei klinischen Populationen, aber auch bei gesunden Kindern und Jugendlichen eingesetzt wird.

Der revidierte KINDL ist ein auf dem Selbstbericht von Kindern und Jugendlichen beruhender Fragebogen mit 24 Items, der sechs Dimensionen der Lebensqualität erfasst (Körper, Psyche, Selbstwert, Familie, Freunde und funktionale Aspekte) und der auf psychometrische Gütekriterien der Reliabilität und Validität überprüft wurde. Es wurden drei Formen des KINDL für verschiedene Altersgruppen entwickelt 
(Kiddy-KINDL für Kleinkinder (vier bis sieben Jahre), Kid-KINDL für Kinder (acht bis zwölf Jahre) und Kiddo-KINDL für Jugendliche (13 bis 16 Jahre)), der Fragebogen ist sowohl in der Selbstberichtsversion als auch in der Elternversion verfügbar. Zusätzlich liegt die Entwicklung einer Kurzform des KINDL (12 Items) vor sowie eine Reihe von krankheitsspezifischen Modulen und verschiedensprachige Übersetzungen (Englisch, Französisch, Holländisch, Russisch, Türkisch, Italienisch und Spanisch).

Der Fragebogen wurde bisher in mehreren Studien an über 3000 gesunden und chronisch kranken Kindern und deren Eltern im Verlauf von bis zu drei Jahren eingesetzt und geprüft. Die psychometrischen Ergebnisse zeigen eine hohe Reliabilität (Cronbach's $\alpha \geq 0.70$ in der Mehrzahl der Skalen und Stichproben) und befriedigende konvergente Validität des Verfahrens an, darüber hinaus besteht eine hohe Akzeptanz des Instruments bei den Kindern und Jugendlichen. Der Fragebogen konnte Kinder mit unterschiedlichen gesundheitlichen Störungen und Belastungsfaktoren differenzieren. Insgesamt erwies sich der KINDL als ein flexibles, modulär aufgebautes, psychometrisch akzeptables Verfahren zur Erfassung der Lebensqualität von Kindern, das sowohl in einem Kernteil generische Aspekte der Lebensqualität von Kindern reflektiert, als auch in Zusatzmodulen die spezifischen Belastungen von Erkrankungen im Kindesalter und die Veränderungen der Lebensqualitätsdimensionen im Entwicklungsverlauf erfasst. Als verbesserungswürdig kann die hohe Skaleninterkorrelationen des Instruments angesehen werden.

\section{Diskussion}

Im vorliegenden Beitrag wurde ein Überblick über die Messmethoden der Lebensqualitätsforschung bei Kindern gegeben. Für die Pädiatrie ist, obwohl sie sich traditionell auch mit psychosozialen Inhalten beschäftigt, die Erfassung von Lebensqualitätsdaten bisher ein eher neuer Bereich, für den neben Fremdbeurteilungsverfahren pädiatrischer Symptomatik auch Selbstbeurteilungsverfahren entwickelt wurden und in dem die soziale Dimension pädiatrischer Erkrankungen zunehmend berücksichtigt wird.
Insgesamt lässt sich ein zunehmendes Forschungsinteresse in der Pädiatrie feststellen, dem aber eine oft nur ansatzweise vorhandene theoretisch-konzeptuelle Auseinandersetzung mit dem Thema der gesundheitsbezogenen Lebensqualitätserfassung bei Kindern verschiedener Altersgruppen und unterschiedlicher chronischer Erkrankungen gegenübersteht. Studien zum Einsatz von Lebensqualitätsmessinstrumenten bei Kindern sind in der Medizin bisher rar. In Bereichen, in denen die Kinder selbst für die Durchführung der Behandlungsmaßnahmen verantwortlich sind, wie z.B. in der Diabetologie oder Asthmatherapie, hat sich eine große Zahl von Untersuchungen mit kindlicher Compliance beschäftigt, die Frage nach Befindlichkeitsstörungen oder Einschränkungen der Lebensqualität ist aber unterrepräsentiert. Fragestellungen, Studiendesigns und verwandte Messinstrumente der Studien sind so heterogen, dass es eine Übersicht erschwert.

\section{„Studien zum Einsatz von Lebensqualitätsmessungen bei Kindern sind in der Medizin noch rar."}

Die zur Erfassung der gesundheitsbezogenen Lebensqualität verfügbaren Instrumente zeigen trotz der großen Anstrengungen und Entwicklungen der letzten Jahre Grenzen, die darin bestehen, dass die Instrumente für Kinder oft zu lang sind und die Entwicklungsperspektive bei der Konstruktion der Instrumente nicht ausreichend berücksichtigt wurde. Es gibt wenig Instrumente, die einen Einsatz in unterschiedlichen Settings, bei verschiedenen Krankheitsgruppen und Schweregraden und in unterschiedlichen sozioökonomischen Gruppen und Kulturen auch im Längsschnitt erlauben.

Der gegenwärtige Forschungsstand im Bereich der Methodenentwicklung erklärt auch die bisher geringe Anzahl von Längsschnittstudien zur Lebensqualität von Kindern und Jugendlichen in der Medizin, speziell in der pädiatrischen Rehabilitation. In Anbetracht der zunehmenden Hinwendung zu psychosozialen Aspekten der Gesundheit, ist der Forschungsbedarf bei Kindern und Jugend- lichen hinsichtlich der subjektiven Gesundheit hoch. Mit Lebensqualitätsmessinstrumenten kann in nationalen Studien die Befindlichkeit und Funktionsfähigkeit großer Bevölkerungsgruppen untersucht werden, die somit sowohl Referenzdaten für erkrankte Kinder als auch Hinweise für medizinischen und gesundheitspolitischen Handlungsbedarf liefern [53, 54]. Im klinischen Kontext geht es um die Verbesserung des Gesundheitszustands von Kindern, nicht nur durch medizinische Verfahren, sondern auch durch Erlernen von psychologischen Strategien [55], was zeigt, dass die Lebensqualität der Kinder hier ein wichtiges Zielkriterium ist.

Voraussetzung für eine positive Entwicklung des Feldes ist die konkrete Definition für die Lebensqualität relevanter Dimensionen bestimmter Patientengruppen in der Pädiatrie, die dann in $\mathrm{Zu}$ sammenarbeit mit sozialwissenschaftlicher Forschung zur Entwicklung von krankheitsspezifischen Lebensqualitätsmessinstrumenten führen kann. Die bisher bestehenden Ansätze sind bereits geeignet, in Forschungsvorhaben einbezogen zu werden, sowohl wegen ihrer bisherigen psychometrischen Fundierung als auch wegen ihrer Funktion als Standardinstrument, an dem neue Entwicklungen geprüft werden können.

In einer von der Division of Mental Health der Weltgesundheitsorganisation (WHO) herausgegebenen Stellungnahme wurden folgende Forderungen an die Eigenschaften von Instrumenten dargelegt:

D das Kind muß im Mittelpunkt stehen (child centred),

D der subjektive Selbst-Bericht hat absoluten Vorrang,

D das Instrument muss altersgerecht oder mindestens der Entwicklungsphase angemessen sein,

D die Ergebnisse müssen interkulturell vergleichbar sein,

D das Instrument sollte aus allgemeinen Kern- (generic core) und spezifischen Modulen (specific modules) zusammengesetzt sein,

D die positiven Aspekte (health enhancing) von Lebensqualität sollten die negativen überwiegen und die verfügbaren Ressourcen der Kinder abbilden.

Um diese Forderungen zu erfüllen, bedarf es einer engen und interdisziplinä- 
ren Zusammenarbeit von Klinikern und Forschern. Wichtig ist, dass für pädiatrische Erkrankungen auch im deutschen Sprachraum mehr Instrumente entwickelt werden und für Kinder zur Verfügung stehen. Eine spezielle Herausforderung der pädiatrischen Lebensqualitätsforschung ist es, den Wert der Selbstbeurteilung der Kinder zu betonen und dabei auch Prozesse der Krankheitsverarbeitung und der Krankheitsbewältigung mit einzubeziehen. Durch die systematische Verbreitung des empirisch fundierten Wissens über die Lebensqualität pädiatrischer Patienten sowie Möglichkeiten ihrer Erfassung und ihrer Veränderung durch therapeutische Strategien kann die Lebensqualitätsforschung in der Kinderheilkunde weiter entwickelt werden. Sie kann dazu beitragen, die Lebensqualität der Kinder nicht nur als Gegenstand der individuellen ArztPatient-Interaktion während der Behandlung, sondern auch als Zielkriterium in klinischen Untersuchungen und Therapiestudien $\mathrm{zu}$ thematisieren. Ein solches Instrumentarium dient letztlich dem Ziel, die Therapieergebnisse erkrankter Kinder und ihrer Familien zu beurteilen und damit Wege der weiteren Verbesserung der medizinischen und psychologischen Betreuung der Betroffenen $z u$ finden.

\section{Literatur}

1. Walker SR, Rosser RM (1991) Quality of life assessment and application. Lancaster, MTP Press

2. Stewart AL, Ware J (1992) Measuring function and well-being. Durham, Duke University Press

3. Ravens-Sieberer U, Bullinger M (1997) Lebensqualität in der Pädiatrie. In: Döhring et al. (Hrsg) Fortschritte in der pädiatrischen Rehabilitation: Qualität und Interdisziplinarität. München, Profil-Verlag

4. Bullinger M (1991) Quality of Life - definition, conzeptualization and implications a methodologists view. Theoretical Surgery 6:143-149

5. Bullinger M, Hasford J (1991) Evaluating quality of life measures in German clinical trials. Controlled Clinical Trials 12:915-1055

6. Stewart AL, Ware J (1992) Measuring function and well-being. Durham, Duke University Press

7. Sartorius N (1987) Cross-cultural comparisons of data about quality of life: a sample of issues. In: Aaronson NK, Beckmann J (eds) The quality of life of cancer patients. New York, Raven Press, pp 1075-1077
8. Bullinger M (1997) Gesundheitsbezogene Lebensqualität und subjektive Gesundheit. Psychother Psychosom Med Psychol 47: 76-91

9. Eiser C, Jenney ME (1996) Measuring symptomatic benefit and quality of life in paediatric oncology. British Journal of Cancer 73:1313-1316

10. Lohaus A (1991) Gesundheit und Krankheit aus der Sicht von Kindern. Göttingen, Hogrefe

11. Bird J,Podmore V (1990) Children's understanding of health and IIIness. Psychology and Health 4: 175-185

12. Bullinger M, Ravens-Sieberer U (1995) Grundlagen, Methoden und Anwendungsgebiete der Lebensqualitätsforschung bei Kindern. Praxis der Kinderpsychologie und Kinderpsychiatrie 10:391-398

13. Neupert $T$, Thorst $G$, Karmaus $W$, Frischer Th, Kopp M, Ulmer Ch, Schwöbel B, Forster J, Kühr J (1997) Asthma und asthmatypische Beschwerden bei Schulkindern:Vergleich von Gebieten in Deutschland und Österreich. Zeitschrift für Gesundheitswissenschaften 5:63-75

14. Lang $S$ (1985) Lebensbedingungen und Lebensqualität von Kindern. Frankfurt, Campus Verlag

15. Seiffge-Krenke I (1990) Krankheitsverarbeitung bei Kindern und Jugendlichen. Jahrbuch der Medizinischen Psychologie. Heidelberg, Springer

16. Wirsching M (1988) Krebs im Kontext. Patient, Familie und Behandlungssystem. Stuttgart, Klett

17. Evans G, Kliener W, Martin J (1991) The role of physical environment in the health and wellbeing of children. In:Schröder $\mathrm{H}$ (ed) New directions in Health Psychology. New York, Hemisphere Press, pp 127-157

18. Neff E, Dale J (1990) Assessment of quality of life in school-aged children. Maternal Child Nursing Journal 19:313-320

19. Barr RD, Pai MKR, Weitzman S (1994) A multiattribute approach to health status measurement and clinical management illustrated by an application to brain tumors in childhood. International Journal of Oncology 4:639-648

20. Ravens-Sieberer U, Theiling St, Bullinger M (1998) Subjektive Gesundheit bei chronisch kranken Kindern und Jugendlichen die Patienten- und die Elternsichtweise. In:Bullinger M, Morfeld M, Ravens-Sieberer U, Koch U (Hrsg) Medizinische Psychologie in einem sich wandelnden Gesundheitssystem: Identität, Integration \& Interdisziplinarität. Lengerich: Pabst Verlag

21. Edelbrock C, Costello AJ, Dulcan MK, Conover NC, Kala R (1986) Parent-child agreement on child psychiatric symptoms assessed via structured inteview. Journal of Child Psychology and Psychiatry 27:181-190

22. Guyatt GH, Junper EE, Griffith LE, Feeny DH, Ferrie PJ (1997) Children and adult perceptions of childhood asthma. Pediatrics 99 : 165-168
23. Juniper EF, Guyatt GN, Feeny DH et al. (1996) Measuring quality of life in the parents of children with asthma. Quality of Life Research 5:27-34

24. Bender BG (1996) Measurement of quality of life in pediatric asthma clinical trials. Annals of Allergy Asthma \& Immunology 77: 438-447

25. Könning J, Szczepanski R, von Schlippe A (1997) Die Betreuung asthmakranker Kinder im sozialen Kontext. Stuttgart: Ferdinand Enke Verlag

26. Harter S,Whitesell NR (1989) Developmental changes in children's understanding of single, multiple and blended emotion concepts. In:Saarni E, Harris PL (eds) Children's understanding of emotion. New York, Cambridge University Press, pp 81-116

27. Pantell RH, Lewis CE (1987) Measuring the Impact of Medical Care on Children. J Chron Dis 187, 40:995-1085

28. Lewis CE, Pantell R, Kieckhefer G (1989) Assessment of Children's Health Status. Medical Care 27:54-65

29. Christie MJ, French D, West A (1991) Childhood Asthma Questionnaire. London: Allen \& Hanbrurys

30. Simmons RG, Blyth DA (1987) Moving into adolescence - the impact of pubertal change and school context. Hawthorne, De Gruyter, NY

31. LaGreca AM, Swales T, Kemp S, Madigan S, Skyler J (1995) Adolescents with diabetes: gender differences in psychosocial functioning and glycemic control. Child Health Care 24:61-78

32. Austin JK, Huster GA, Dunn DW, Risinger MW (1996) Adolescents with active or inactive epilepsy or asthma: a comparison of quality of life. Epilepsia 37:1228-1238

33. Ravens-Sieberer U, Bullinger M (1998) Quality of Life from a Patient Group Perspective. In: Koot H, Wallanger M (eds) Quality of Life in Children and Adolescents: Concepts, methods and findings. (im Druck)

34. Eiser C (1997) Children's quality of life measures. Archives of Diseases in Childhood 77: 350-354

35. Landgraf I, Abetz L, Ware J (1997) Child Health Questionaire (CHQ): a users manual. Boston, The Health Institute Press

36. Granger CV, Gresham GE (1984) Functional Assessment in Rehabilitation Medicine. Baltimore, William \& Wilkins

37. Haley SM, Coster WJ, Ludlow LH (1991) Pediatric functional outcome measures. Physical Medicine and Rehabilitation Clinics of North America 2:689-723

38. Achenbach TM, Edelbrock C (1983) Manual for the Child Behavior Checklist and Revised Child Behavior Profile. University of Vermont, Dept. of Psychiatry, Burlington

39. Lewis CE (1981) Measuring the well-being of children. Child Health 42:120-125

40. Bullinger M, Ravens-Sieberer U (1995) HealthRelated Quality of Life Assessment in Children:A Review of the Literature. Euro Rev Appl Psychol 45:245-254 
41. Landgraf JM, Ravens-Sieberer U, Bullinger U (1997) Quality of life research in Children: methods and instruments. In:Gerharz EW (ed) Dialogues in Pediatric Urology 20:5-7

42. Ravens-Sieberer U, Bullinger M (1998) Quality of Life from a Patient Group Perspective. In: Koot HM, Wallanger (eds) Quality of Life in Children and Adolescents: Concepts, methods and findings (im Druck)

43. Landgraf JM, Ware JE, Schor E, Ross-Davies A, Rossi-Roh K (1993) Comparision of health status profiles for children with medical conditions preliminery psychometric and clinical results from children's health and quality of life project. Paper prepared for the 10th anual meeting for Health Services Research, Washington

44. Landgraf JM, Ravens-Sieberer U, Bullinger M (1997) Quality of life research in Children: methods and instruments. In:Gerharz EW (ed) Dialogues in Pediatric Urology 20:5-7

45. Verrips GH, Vogels AGC, Verloove-Vanhorick, SP et al. (1997) Health-related quality of life measure for children - the TACQOL. J Appl Therapeutics 1:357-360

46. Bruil J, Maes S, le Coq L, Boeke J (1996) The development of the how are you (HAY), a qality of life questionnaire for children with a chronic illness. Quality of Life Newsletter 13:9

47. Simeoni MC, Auquier $P$, Gentile S, Jouve E, San Marco JL (1998) Results of the conceptualisation and validity of a new French health related quality of life instrument in adolescence. Paper presented at the 5th Annual Conference of the International Society for Quality of Life Research, Balitmore

48. Manificat S, Dazord A (1998) Children's quality of life assessment: preliminary results obtained with the AUQUEI questionnaire. Quality of Life Newsletter 15:2-3
49. Starfield B, Riley A (1998) Profiling heath and illness in children an adolescents. In: Drotar D (ed) Measuring Health-Related Quality of Life in Children and Adolescents. New Jersey: Implications for Research and Practice. Lawrence Erlbaum

50. Grob A, Luthi R, Kaiser F, Flammer A, Mackinnon A, Wearing A (1991) Berner Fragebogen zum Wohlbefinden Jugendlicher (BFW). Diagnostica 37:66-75

51. Bullinger M, Mackensen S, Kirchberger I (1994) KINDL - ein Fragebogen zur gesundheitsbezogenen Lebensqualität von Kindern. Zeitschrift für Gesundheitspsychologie 2: 64-67

52. Ravens-Sieberer U, Bullinger M (1998b) Assessing the health related quality of life in chronically ill children with the German KINDL: first psychometric and contentanalytical results. Quality of Life Research 7: 399-408

53. Lang $S$ (1985) Lebensbedingungen und Lebensqualität von Kindern. Frankfurt, Campus Verlag

54. Lindström B, Eriksson B (1993) Quality of life among children in the Nordic Countries. Quality of Life Research 2:23-32

55. Compas BE (1987) Coping with Stress during childhood and adolescence. Bulletin 101:393-403

56. Maylath NS (1990) Development of the Children's Health Rating Scales. Health Education Quarterly 17:89-97

57. Boyle MHJ, Offord DR, Racine VA, Fleming JF (1993) Evaluation of the revised Ontario Child Health Study Scales. J Child Psychol Psych Allied Discipl 34: 189-213

58. Feeny DH, Torrance GW, Furlong WJ (1996) Health Utilities Index. In:Spilker B (ed) Quality of life and Pharmaeconomics in clinical trials Philadelphia. Philadelphia:LippincottRaven, pp 239-252

\section{J. Haisch, R. Weitkunat, M. Wildner (Hrsg.) Wörterbuch Public Health}

Bern/Göttingen/Toronto/Seattle: Verlag Hans Huber, 1999. 486 Seiten, (ISBN 3-456-83051-3), geb., DM 49,80

ABDA, DMP, Gompertzsches Gesetz, Spleen Rate...Wissen Sie was gemeint ist? Eine wertvolle Hilfe liegt nun vor zur raschen Orientierung in der schier babylonischen Sprachverwirrung, die bei der dynamischen Entwicklung der multidisziplinären Gesundheitswissenschaften wohl unausbleiblich ist. Dem Wörterbuch mit knappen Erläuterungen zu rund 4000 Begriffen aus dem weiten Feld von Public Health messen die Autoren in sympathischer Weise selbst „Werkstattcharakter" zu. Daher werden die Benutzer zur Mitwirkung aufgerufen bei der geplanten Fortschreibung. In einem ausführlichen Serviceteil finden sich zudem Hinweise zu weiterführender Literatur, zu PHrelevanten Zeitschriften und Internetadressen; ferner Adressen und weitere Angaben zu PHStudiengängen, zu entsprechenden FH-Angeboten, Seminaren, Instituten u.a.m. Das Wörterbuch ist allen im Bereich der Öffentlichen Gesundheit Beschäftigten und darüber hinaus allen daran Interessierten zu empfehlen. Dem Buch - sein Inhalt ist ergänzend auch als CD im HTML-Format für DM 10,- erhältlich (Bestellkarte im Serviceteil) - ist eine rasche und weite Verbreitung zu wünschen.

G. Kerscher (München) 